\title{
Orden básico de constituyentes en construcciones transitivas sintácticas del español actual
}

\section{Basic Order of Constituents in Transitive Syntactic Constructions in Contemporary Spanish}

Javier Puerma Bonilla [javierpuermabonilla@gmail.com]

Universidad de Granada, España

\section{RESUMEN}

Este trabajo analiza y describe el orden básico de constituyentes en construcciones transitivas del español actual. El objetivo central es estudiar el orden relativo entre verbo transitivo y objeto directo léxico, vo vs. ov. Los cuatro tipos de prosa rastreados tienen la función de favorecer contextos discursivos diversos que propicien o inhiban la alternancia en el orden. Finalmente, el sujeto, léxico o morfológico, ha sido tomado en cuenta como variable de análisis para observar el comportamiento del orden relativo entre verbo transitivo y objeto directo léxico.

\section{Palabras Clave}

orden; constituyentes; oD léxico; género textual

\begin{abstract}
This paper analyses and describes the basic order of constituents in syntactic constructions of $21^{\text {st }}$ century Spanish. The aim of the paper is to study the relative order between the transitive verb and the lexical direct object, vo vs. ov. The four types of prose tracked seek to favour different discursive contexts that either promote or inhibit the shift in the order. Finally, the subject, either lexical or morphological, has been taken into consideration as a variable in order to observe the performance of the relative order between the transitive verb and the lexical direct object.
\end{abstract}

\section{KEYWORDS}

Order; constituents; lexical Do; literary genre

RECIBIDO 2018-10-26; ACEPTADO 2019-01-03 


\section{Introducción}

En este trabajo, analizaré las construcciones transitivas con objetos directos (en adelante oD) léxicos, integrados por una frase nominal (en adelante FN) o por una frase preposicional (en adelante FP), en aquellos oD léxicos encabezados por la preposición $a$, cuyo núcleo sea un sustantivo (1a-d) o un pronombre $(2 \mathrm{a}-\mathrm{d})$. A continuación, marco el verbo transitivo en negrita y cursiva, y el oD léxico en negrita.

1a. Los neandertales y los hombres modernos representan dos trayectorias evolutivas distintas e independientes [Bartra, 31, xxI]

1b. Los eventos críticos incrementan la tensión dentro del grupo familiar [Medicina, 37, xxI]

1c. La competencia sin merecimiento no necesariamente produce autoestima [Medicina, 20, xxI]

1d. Al igual que Humphrey Velmans, Edelman esquiva el asunto al que nos han enfrentado el autismo y los comportamientos antisociales [Bartra, 90, xxI]

2a. pero al Toto ya no lo vi, que donde apareció muerto yo vi que había varios con él y lo vi de retirado, estaban como unos cuatro o cinco con él [DLXXI, 25, XXI]

2b. Es que la laboriosidad es el germen de toda fealdad, pero no he dejado de tener ideas y, es más, si quieres te vendo una [Bartleby, 46, xxI]

2c. En segundo lugar, existe un elemento afectivo, una valencia o grado de naturaleza positiva o negativa adherida a esas facetas identificadas, llamamos a esto alta o baja autoestima [Medicina, $15, \mathrm{XxI}]$

2d. Antes del día del estreno y también en los días que siguieron a éste, a lo largo de su último mes de vida, Wilde entendió que una extensión de su felicidad podía darse - la obra en Londres estaba teniendo un gran éxito- en la sistemática petición de más royalties por la obra estrenada en el Royalty [Bartleby, 46, xxi]

El orden básico de constituyentes ${ }^{1}$ se ha erigido históricamente en una de las primeras y principales preocupaciones de la sintaxis en lengua española, como atestigua Nebrija en la Gramática sobre la lengua castellana (1492/2015: cap.IV), al afirmar que "entre algunas partes dela oración ai cierta orden casi natural i mui conforme a la razón, enla cual las cosas que por naturaleza son primeras o de maior dignidad, se an de anteponer a las siguientes et menos dignas". En otras palabras, dentro de la estructura oracional los diferentes elementos gramaticales que la componen están distribuidos jerárquicamente. Por su parte, Bello (1847-1860/1988: \$478) da cuenta de la estructura de la oración y explica que "Habiéndose dado a conocer [...] los varios elementos de que se compone la oración, es ya tiempo de manifestar el orden y dependencia en que los colocamos".

El objetivo principal de esta investigación es analizar el orden de constituyentes en construcciones transitivas sintácticas con od léxicos. Como es sabido, el español admite dos órdenes relativos

1 Para los fines de esta investigación, emplearé la etiqueta de cobertura orden básico de constituyentes en lugar del muy difundido orden de palabras, puesto que, en rigor, este es el objeto de estudio de la investigación, no así el orden entre cualquier clase de palabras. Esta argumentación es compartida por Comrie (1981/1989: 86), quien advierte que "aunque mantenemos el término orden de palabras [...], el cual se ha establecido para hacer referencia a esta área de la tipología [...], en sentido estricto, nos ocupamos no tanto del orden de palabras como del orden de constituyentes". 
posibles entre verbo transitivo y oD léxico: $i)$ orden vo (3a-d), y ii) orden ov $(4 a-d)^{2}$. Ejemplifico ambos órdenes abajo.

3a. Pero no fue allí sino en Puerto Vallarta donde por primera vez alguien me contó la historia de Traven [Bartleby, 71, xxI]

3b. Ian Tattersall encuentra la clave en la llamada exaptación [Bartra, 33, xxI]

3c. Por una parte, el estatus implica estados del ser, por otra parte los estatus deben mantenerse [Medicina, 20, xxi]

3d. De los elementos expuestos, valorados conforme a lo estatuido en el artículo 453, del ordenamiento penal invocado, permite establecer tienen derecho a la reparación de los daños y perjuicios respecto de la víctima José Guadalupe Martínez Rodríguez [DLXXI, 47, XXI]

4a. Se consigna ahí que Alonso Dávila compró un navío a un Hernando Martínez, "que es uno que vino en la armada”, el cual pagó Cortés [Hernán Cortés. Inventor de México, 87, México, 2001, CREA]

4b. Fueron tantas cosas y tan locas, que casi no me acuerdo, ese período lo tengo como borrado [Yo soy el Diego, 272, Argentina, 2000, CREA]

4c. Nos visitan en la enfermería algunos compañeros que salen a secar su ropa al sol o que van al comedor. A muchos no los conozco pero me demuestran simpatía y amistad [Cómo llegó la noche. Revolución y condena de un idealista cubano, 485, Cuba, 2002, CREA]

4d. me fui a mi casa con una botella de mezcal curada con refresco, pero al Toto ya no lo vi [DLXXI, $25, \mathrm{xxI}]$

Este trabajo sobre orden de constituyentes en construcciones transitivas es novedoso porque se apoya en los datos extraídos de un corpus sincrónico del español, los cuales tienen en cuenta tanto la tipología textual, como variedades del español peninsular y americano; esto es especialmente interesante, puesto que este estudio pretende ampliar el enfoque más acotado, por razones temporales, dialectales y textuales, de otros estudios. Esta investigación aporta dos nuevas informaciones. Por un lado, prueba documentalmente que, de acuerdo con los datos analizados, en el xxI el orden vo del español no solo es mayoritario, sino casi absoluto. Por otro lado, aunque sabemos que el español es descrito unas veces como lengua svo, otras veces como lengua vo, este trabajo describe el papel del sujeto en el orden básico.

Existen, además, dos informaciones relevantes: una de naturaleza sintáctico-pragmática y otra de naturaleza teórica. Sabemos que el orden de constituyentes permite a los hablantes empaquetar y jerarquizar la información, priorizarla o postergarla, mediante la codificación en elementos gramaticales de manera secuencial. En este sentido, el español, como lengua no flexiva, exhibe un comportamiento similar al de otras lenguas románicas, como el francés o el italiano, en la disposición vo de sus elementos, aunque este orden se presente en cada una de estas lenguas con diferentes grados de rigidez estructural. No obstante, en el español parecen convivir, como sostienen algunos autores (Elvira 2013: 209), la rigidez gramatical de la pauta vo y cierta flexibilidad

2 Para aquellos casos carentes de documentación en el corpus base de esta investigación emplearé ejemplos del Corpus de Referencia del Español Actual (CREA) 
heredada del latín. Esta información refuerza el interés de este trabajo por cuanto, al contrario de lo que sostienen los trabajos especializados, el orden de constituyentes parece tender a una cierta rigidización ${ }^{3}$ en la actualidad, al menos, en lo que respecta al español escrito del corpus estudiado ${ }^{4}$. En línea con lo anterior, los resultados del corpus son interesantes, porque, si bien es cierto que en español coexisten dos tipos de órdenes distintos, vo vs. ov, el siglo xxi supone no solo la constatación de que el orden vo es mayoritario, sino que los datos sugieren que el español se decanta por un orden sintáctico vo casi absoluto en detrimento de posibles fluctuaciones por razones pragmáticas, discursivas o de género textual. Lo anterior supone, a mi juicio, una información novedosa respecto del estudio del orden básico de constituyentes en español actual. Finalmente, aunque es bien conocido que las lenguas son filtros de arbitrariedad y simbolismo, el orden de constituyentes parece reproducir fenómenos de iconicidad a nivel sintáctico, puesto que la estrecha vinculación de verbo transitivo y oD léxico se refleja en la posición relativa que ambos constituyentes guardan entre sí.

Este tema sobre orden de constituyentes en construcciones transitivas del español actual ha pasado relativamente inadvertido, señal de que han sido otros los fenómenos de la gramática que han suscitado mayor preocupación. Si bien es cierto que el orden de palabras en general ha sido un tema ampliamente tratado en los siglos XIX y Xx, hasta donde sé, no existen numerosos estudios que aborden la distribución relativa entre verbo transitivo y oD léxico en el español del siglo xxI con base en un corpus sincrónico que tome en cuenta la tipología textual y el español general. Precisamente, este trabajo tiene como objetivo primordial realizar este análisis.

El objetivo específico de este trabajo es describir el orden relativo entre verbo transitivo y oD léxico con base en cuatro tipos de prosa, que propicien contextos comunicativos diversos. Haré este análisis atendiendo a si este orden básico u orden preferido lo es en términos relativos o absolutos, esto es, si la diversidad textual propicia o inhibe ciertos órdenes, o si no lo hace en absoluto. Finalmente, aunque el sujeto no forma parte de nuestro objeto de estudio, lo tomaré en cuenta como variable de análisis, esto es, para evaluar si la presencia o ausencia léxica del mismo condiciona la distribución del on respecto del verbo transitivo.

Esta investigación se compone de la presente introducción, así como de otros seis apartados. En $\$ 2$, expondré el corpus que guía este artículo; en $\$ 3$, abordaré los aspectos más relevantes respecto del orden básico de constituyentes de verbos transitivos del español, tratados por la bibliografía general y especializada hasta la fecha, prestando especial atención al español actual. En $\$ 4$, analizaré la persistencia del sujeto léxico en términos de presencia o ausencia en relación al género textual. En \$5, abordaré el orden de constituyentes en construcciones transitivas del español actual con sujeto léxico o explícito y oD léxico, y con sujeto morfológico o ausente y oD léxico. En $\$ 6$, terminaré con unas conclusiones.

3 Esta rigidización sintáctica, como argumenta en un reciente estudio Puerma Bonilla (2018: 91-111), está acompañada por una rigización informativa. La función de tópico, asumida mayoritariamente por el sujeto léxico desde las primeras etapas del español, se ha extendido diacrónicamente, y se ha generalizado respecto de este constituyente argumental en el siglo Xxi.

4 Puerma Bonilla (en proceso) documenta una mayor alternancia entre los diversos órdenes de constituyentes del español en un estudio diacrónico. 


\section{Corpus}

La presente investigación abarca la primera década del siglo xxi. Con el fin de dotar al análisis de mayor dinamismo, los datos cuantitativos y cualitativos han sido extraídos de un corpus con base en cuatro tipos distintos de prosa: prosa literaria, prosa ensayística e historiográfica, prosa jurídica y prosa científica. La diversa tipología textual tiene como objetivo proveer a las construcciones transitivas estudiadas de variados contextos discursivos, donde las necesidades pragmáticas se manifiestan, propiciando o inhibiendo determinados órdenes de constituyentes.

Para los fines de esta investigación entenderé, en primer lugar, por prosa literaria aquella cuyos rasgos característicos son la ficción y la creación; en segundo lugar, entenderé por prosa ensayística e historiográfica aquella cuyos rasgos definitorios son la documentación, la divulgación, la reflexión y el análisis; en tercer lugar, entenderé por prosa jurídica aquella que versa sobre asuntos legales o administrativos, de carácter formulaico, y estructura en forma de declaración y dotada de cierta solemnidad; en cuarto y último lugar, la prosa científica expone hechos objetivos, que son susceptibles de ser comprobados.

Finalmente, los textos en prosa que conforman el corpus objeto de estudio y sus abreviaturas son: Bartleby y compañía [Bartleby]; Antropología del cerebro [Bartra]; Documentos jurídicos XXI [DLXxi]; y Compendio de tesis de medicina [Medicina ${ }^{5}$.

En relación al establecimiento del fichado en los cuatro géneros textuales, he realizado un muestreo al azar en un corpus con base en un total de 16000 palabras por obra ${ }^{6}$. El número total de palabras analizadas está determinado por la alta ocurrencia de casos documentados de transitividad sintáctica. Por su parte, en este trabajo analizo el español general, en concreto variedades de España y México, por lo que están representados tanto el español de España como el de América Latina.

En relación con la metodología que ha guiado la conformación del corpus, estudio od léxicos, integrados por una FN o una FP encabezada por la preposición $a$, cuyo núcleo puede ser un sustantivo o un pronombre. Con el fin de estabilizar formalmente el análisis, he excluido del fichado los oD clíticos, oracionales, coordinados, así como los compuestos por formas no personales del verbo. Respecto de las características léxico-semánticas de los od léxicos, he incluido en el corpus nombres propios y comunes, oD humanos y no humanos, simples y no simples.

\section{Breve estado de la cuestión}

El orden básico de constituyentes presenta cuatro ejes bien definidos, algunos de los cuales resultan, al mismo tiempo, polémicos, puesto que los resultados arrojados por la presente investigación matizan algunos de los aspectos que han sido tratados con anterioridad. Cabe destacar que es infrecuente encontrar estudios que aborden el orden básico de constituyentes del español actual y

5 Para ver la bibliografía completa de las mismas, consúltese el apartado $₫ 7$.

6 He mantenido el mismo universo de palabras para cada obra analizada, puesto que dicho universo da cuenta de una información hasta donde sé novedosa, además del orden preferido y de la presencia o ausencia léxica del sujeto: la diferente productividad de la transitividad según el género textual. Así, los textos que han registrado un mayor número de construcciones transitivas pertenecen a la prosa novelada y a la prosa ensayística e historiográfica, y los que menos, a la prosa científica y a la prosa jurídica. 
general, con diversidad de tipología textual, como señalé arriba, por lo que la mayor parte de los estudios encontrados suelen estar acotados por limitaciones de índole temporal, textual, dialectal o sociolingüística, o a aspectos determinados del orden de constituyentes en construcciones transitivas con verbos de una determinada clase verbal.

Los ejes que guían este breve estado de la cuestión son: a) Estatus sintáctico y semántico del sujeto y del objeto directo en construcciones transitivas; $b$ ) Orden básico de constituyentes en español; $c$ ) Orden de constituyentes en la tipología; $d$ ) Orden de constituyentes y tipos de verbos.

a) Estatus sintáctico y semántico del sujeto y del objeto directo en construcciones transitivas Es sabido que el español, en calidad de lengua pro-drop, puede prescindir del sujeto léxico bajo determinados condicionantes. Así, para Elvira (2012) el sujeto constituye el "argumento más alto o externo" dentro de la construcción. En este sentido, el sujeto es considerado por el autor (2011) como un elemento secundario respecto de la construcción transitiva, que solo puede ser definido de manera adecuada en relación a la construcción transitiva. Finalmente, Lehmann (1973: 51-65) ya había sostenido con anterioridad que los "sujetos, de ninguna manera, son elementos primarios en las oraciones".

Para algunos autores (Rodríguez Ramalle 2005: 201-202; Elvira 2012: 8) los papeles semánticos ejercidos por el sujeto conocen pocas restricciones: agente, causa, instrumento, experimentante, beneficiario, paciente, tema. Para Elvira (2015: 207-211), la primera posición corresponde a elementos que desempeñan el papel semántico de tópico; así, identifica al sujeto gramatical, precisamente por su "mayor disponibilidad para desempeñar el papel informativo de tópico" como candidato idóneo para ocupar esa primera posición. Bogard (2015: 34-36) encuentra que, para el siglo xIx, el sujeto está "omitido", y cuyo referente tiene un "elevado valor topical" (cf. Givon 1976; apud Bogard 2015), asimismo, denomina al sujeto "el objeto de la comunicación". Ordóñez y Treviño (1999) consideran al sujeto "un tópico por defecto". Finalmente, Gutiérrez Bravo (2007) argumenta que la posición inicial no está motivada por el caso, la concordancia o por ser el sujeto un tópico por defecto, sino que "las cláusulas del español con diferentes tipos de constituyentes en posición preverbal poseen diversos grados de marcación", y que "ese grado de marcación depende del papel semántico del constituyente preverbal" (cf. Prince y Smolensky 2004; apud Gutiérrez Bravo).

En lo tocante al od, Elvira (2012: 1) le reserva el estatus de "argumento más bajo o interno". Además, el autor (2011) considera al oD un elemento secundario respecto de la construcción transitiva, que solo puede entenderse de manera adecuada cuando se encuentra definido en relación a la construcción a la que pertenecen. Algunos autores (Campos 1999: 1531-1539; Elvira 2012: 8) argumentan que los papeles semánticos desempañados por el oD son mucho más restrictivos, reduciéndose a paciente, si está afectado, o tema, si no lo está.

b) Orden básico de constituyentes en español

Existen numerosos trabajos que identifican al español como una lengua svo (cf. Contreras 1983; Silva-Corvalán 1984; Bentivoglio y Weber 1986; Brucart y Hernanz 1987; Ocampo 1990; Gutiérrez Bravo 2007; Fernández Soriano 2011; Elvira 2015). En este sentido, Elvira (2015: 207-211) sostiene que se ha producido a lo largo de la historia del español un cambio del antiguo orden sov 
a un moderno svo, y contrasta el dinamismo informativo y pragmático del antiguo sov con un nuevo orden svo más rígido "regulado esencialmente por principios gramaticales". Por su parte, Bogard (2015: 34-36) establece, en primer lugar, cierta ambivalencia ${ }^{7}$ en cuanto a la alternancia de los órdenes svo y vso en el siglo xIII. Sin embargo, encuentra que, en el siglo xix, la oración transitiva más frecuente del español sigue un orden vo, donde el sujeto está "omitido". En segundo lugar, el autor (2010: 73) ${ }^{8}$ identifica el orden vo como "dominante" o no marcado; el orden svo como orden "marcado"; y en otros ${ }^{9}$ registra el resto de órdenes ${ }^{10}$ posibles. Por otro lado, Gutiérrez Bravo (2007: 236) sugiere que los órdenes vso y vos son agramaticales. No obstante, otros autores (Contreras 1991; Alexiadou y Anagnostopoulou 1998; apud Gutiérrez Bravo 2007) apuntan a que el español parece alternar los órdenes svo y vso, aunque el primero tiende a prevalecer sobre el segundo. En un análisis sociolingüístico del español mexicano, Martín Butragueño (1997: 511-532) demuestra que, con independencia del estrato social ${ }^{11}$, el orden vo documenta $70 \%$ de casos, mientras que el orden ov solo $30 \%$. A su vez, en un análisis sobre la adquisición del orden de constituyentes del español en niños de cinco, siete y nueve años, que son bilingües en quechua y español, Luján, Minaya y Sankoff (1984; apud Ocampo y Klee 1995) encuentran que el quechua interfiere en la adquisición del orden de constituyentes en español ${ }^{12}$.

\section{c) Orden de constituyentes en la tipología}

Weil (1844/1978) y Schmidt (1926) son los primeros en estudiar el orden de constituyentes. Greenberg (1963/1966: 73-113) concluye en un estudio de "naturaleza tentativa" que los órdenes de constituyentes posibles son seis: svo, sov, vso, vos, osv y ovs; pero de ellos, los tres últimos (vos, osv y ovs) son especialmente infrecuentes, y se caracterizan por preceder siempre el oD al sujeto. Comrie (1981/1989: 87) establece dos órdenes dominantes entre las seis combinaciones posibles: svo y sov. Además, el autor señala algo muy interesante y es que en lenguas con un orden rígido de constituyentes, como el inglés, conviven diferentes órdenes adscritos a tipos de oraciones particulares, como las interrogativas. Por su parte, Lehmann (1973: 51-65) propone reducir todos los órdenes posibles a dos: vo y ov. El autor (Lehmann 1973: 55) clarifica que "cuando las lenguas muestran patrones diferentes a los esperados, debemos entender que se encuentran en proceso

7 El autor documenta 35 oraciones (54\%) con orden svo, y 30 oraciones (46\%) con orden vso.

8 Analiza tres obras pertenecientes a la última década del siglo xx, y a la primera década del siglo xxI; es un estudio diatópico con textos procedentes de México, Colombia, y España.

9 En otros incluye el orden ov.

10 Los datos documentados para el orden vo son: México (73.4\%), Colombia (69.8), y España (66.8); para el orden svo son: México (24.3\%), Colombia (28.2\%), y España (31.0\%); y para otros son: México (2.3\%), Colombia (2.0\%), y España (2.2\%).

11 Para su investigación, el autor ha conformado un corpus donde se centra en el análisis de la prosa periodística mexicana: estrato alto: Uno más uno; estrato medio: El Universal; y estrato bajo: La Prensa. Así, Martín Butragueño aclara que es, en efecto, cuestionable si los tres periódicos utilizados corresponden con exactitud a los estratos sociales identificados.

12 Los autores analizan tres grupos de tres usuarios bilingües de quechua y español cada uno; el primer grupo aglutina usuarios de cinco años; el segundo grupo, de siete años; y el tercer grupo, de nueve años. Para el grupo de cinco años se encuentra un orden relativo entre verbo y oD ambivalente: $51 \%$ (ov), y $49 \%$ (vo); el grupo de siete años documenta $40 \%$ de casos (ov), y $60 \%$ (vo); por último, el grupo de nueve años registra 30\% de casos (ov), y $70 \%$ (vo). En suma, mientras que en los usuarios de cinco años el orden relativo entre verbo y oD es ambivalente, con el paso del tiempo el orden vo se hace claramente mayoritario, por lo que este estudio parece confirmar la interferencia interlingüística en usuarios bilingües de quechua y español. 
de cambio"; de este modo, durante este período de transición las lenguas no pueden considerarse vo ni ov, por lo que las denomina ambivalentes (Lehmann 1972: 989). Finalmente, Vennemann (1973: 40-41) propone el principio de serialización natural, basado en el principio de estructura natural de constituyentes, desarrollado por Barstch. Este principio dice que "los elementos que están unidos en la jerarquía de representación semántica tienden a lexicalizarse y serializarse en la representación de superficie de manera que las dependencias jerárquicas se reflejan directamente en relaciones categoriales operador-operando", y se serializan de manera unidireccional: $\{[[\mathrm{Ope}-$ rando] Operador]\}, en que el operando es el verbo y el operador el oD, para lenguas con orden vo como es el español.

d) Orden de constituyentes y tipos de verbos.

Lehmann (1973: 51-65) identifica al verbo como el "elemento fundamental en la ordenación de los elementos gramaticales". Esto le lleva aducir que el orden relativo entre verbo y oD es fundamental, por lo que el orden de los demás elementos depende directamente de este (cf. Greenberg 1963). Para Elvira (2012: 1) los argumentos están organizados en dependencia directa del tipo de verbo, en otras palabras, evidencia "la existencia de regularidades en la forma de organizar los argumentos que tienen significados similares"; así, en la organización argumental y sus regularidades varían según la clase verbal. En concreto, Gutiérrez Bravo (2007: 236) argumenta que distintas clases semánticas de verbos parecen requerir otros órdenes no marcados, refiriéndose en particular, a los verbos psicológicos.

\section{Presencia vs. ausencia del sujeto léxico en construcciones transitivas del español actual y género textual}

En este apartado analizaré la persistencia del sujeto gramatical en términos de presencia y ausencia léxica con un doble propósito. Por un lado, estableceré el comportamiento sintáctico del sujeto en construcciones transitivas, para determinar en qué grado este está documentado como parte esencial de las construcciones con verbo transitivo en el siglo xxi y, por tanto, si guía la presente investigación. Por otro lado, procederé a cotejar los resultados obtenidos con el género textual, con el objetivo de poner de manifiesto si el comportamiento del sujeto en construcciones transitivas es homogéneo o si, por el contrario, muestra asimetrías según el soporte textual que lo documenta. Para ello, analizaré las construcciones con sujeto léxico o explícito (5a-d) y con sujeto morfológico o ausente (6a-d) por género textual. Los sujetos presentes están integrados por FN simples y no simples, pronombres, nombres comunes y propios, concretos y no concretos. En los ejemplos abajo marco el sujeto léxico o explícito en negrita y el verbo en cursiva.

5a. Porque no me cabe duda de que sus notas mitifican el tema del silencio en la escritura [Bartleby, 60]

5b. Se podría decir que el exocerebro sustituye el desorden de la confrontación con una diversidad de nichos ecológicos por el orden generado por un nicho simbólico estable [Bartra, 32]

5c. Por una parte, el estatus implica estados del ser, por otra parte los estatus deben mantenerse [Medicina, 20] 
5d. de momento yo no decía nada de lo que había pasado, porque se encontraban mis familiares $[D L X X I, 27]$

6a. En cuanto aprendes inglés empiezan las complicaciones. Por mucho que lo intentes, siempre llegas a esta conclusión [Bartleby, 6]

6b. Para sobrevivir utiliza nuevos recursos que se hallan en su cerebro [Bartra, 32]

6c. por autoestima entendemos la evaluación que efectúa y mantiene comúnmente el individuo en referencia a sí mismo [Medicina, 16]

6d. al ir caminando yo aproximadamente dos metros adelante de mi hijo, de pronto me agaché hacia el piso y tomé una piedra de las "brutas" que era más chica que mi mano [DLXXI, 26]

Los trabajos encontrados sobre orden de constituyentes, expuestos en el estado de la cuestión, ponen en entredicho la obligatoriedad del sujeto léxico o explícito como parte integral de la construcción. Por lo que cabría esperar que los datos del corpus confirmen esta hipótesis.

\section{Cuadro 1}

Presencia vs. ausencia del sujeto léxico

$\begin{array}{lll} & \text { presencia } & \text { ausencia } \\ \text { Bartleby } & 35 \%(33 / 95) & 65 \%(62 / 95) \\ \text { Bartra } & 58 \%(48 / 83) & 42 \%(35 / 83) \\ \text { Medicina } & 71 \%(35 / 49) & 29 \%(14 / 49) \\ \text { DLXXI } & 43 \%(23 / 54) & 57 \%(31 / 54) \\ & & \\ \text { PROMEDIO } & 49 \%(139 / 281) & 51 \%(142 / 281) \\ \mathrm{N}=281 & & \end{array}$

En general, los datos evidencian que las construcciones transitivas del español actual son ambivalentes en cuanto a la presencia, con $49 \%$ de casos, y ausencia, con $51 \%$, del sujeto léxico o explícito. Esta información resulta muy significativa y subraya la importancia del constituyente sujeto como integrante, de al menos, la mitad de construcciones transitivas del español actual, por lo que resulta pertinente considerarlo como parte esencial de este análisis.

No obstante lo anterior, sorprende la claridad con que el género textual, como macrolocus ${ }^{13}$ del cambio sintáctico (Company 2016), condiciona la presencia o ausencia del sujeto léxico o explícito. En este sentido, encontramos, por un lado, que Medicina, con 71\%, y Bartra, con 58\%, prefieren sujetos léxicos o explícitos; y, por otro lado, que Bartleby, con 35\%, y DLXXI, con 43\%, prefieren sujetos morfológicos o ausentes. Resulta significativo que el texto con un mayor grado de especialización posea mayor porcentaje de sujetos léxicos o explícitos, y el texto más creativo registre el mayor porcentaje de sujetos morfológicos o ausentes.

13 El género textual actúa como agente, propiciando o inhibiendo el cambio sintáctico. 


\section{Orden de constituyentes en construcciones transitivas del español actual}

A lo largo de este apartado, analizaré el orden básico de constituyentes en construcciones transitivas del español actual, tanto con sujetos léxicos (\$5.1), como con sujetos morfológicos (\$5.2). Finalmente, terminaré con la exposición de la diacronía general del orden de constituyentes, independientemente del tipo de sujeto consignado en las construcciones transitivas encontradas en el corpus base.

\subsection{Orden básico de constituyentes con sujeto léxico}

En este apartado estudiaré el orden básico de constituyentes en construcciones transitivas con sujeto léxico o explícito ${ }^{14}$ y od léxico (7a-d). Para los fines de esta investigación y en consonancia con los datos consignados en el presente corpus del español actual, analizaré las construcciones transitivas con sujeto léxico o explícito para evaluar si la presencia de un sujeto léxico o explícito condiciona el orden relativo entre verbo transitivo y od léxico. A continuación, muestro ejemplos paradigmáticos de construcciones transitivas con sujeto léxico o explícito, y od léxico; marco el sujeto léxico o explícito en cursiva, y el od léxico en negrita.

7a. Porque no me cabe duda de que sus notas mitifican el tema del silencio en la escritura [Bartleby, 60, xxi]

7b. La pequeña Helen tenía ciertos rasgos autistas que sin duda provenían de la ausencia de lazos sociales importantes [Bartra, 126, xxI]

7c. el hoy occiso tuvo seis hermanos de nombres Alberto, José de Jesús, Daniel, Cecilia, Yesenia y Maibeline [DLXXI, 47, XXI]

7d. Gran parte de los trastornos psicosociales tienen su origen en la disfunción familiar [Medicina, $37, \mathrm{xxI}]$

En relación a los datos mostrados en (7) arriba, cabe destacar que en el siglo xxi, tanto sujetos léxicos o explícitos como od léxicos se caracterizan por estructuras internas de frase nominal con un contenido considerable de elementos gramaticales a ambos lados del núcleo, esto es, modificadores, a la izquierda del núcleo, y expansiones a la derecha del mismo (Company 1991: cap. 1; apud Company 2009). Esto debe tomarse en consideración, puesto que parece que a mayor amplitud de la estructura interna de la frase nominal, más se robustece el orden básico de constituyentes en construcciones transitivas del español actual vo, o, cuando el sujeto está presente como en este caso, un orden básico de constituyentes en construcciones transitivas del español actual svo.

14 El estudio pormenorizado de este corpus exige el análisis del orden básico de constituyentes en construcciones transitivas desde dos ángulos bien diferenciados: con sujeto léxico o explícito, por un lado; y con sujeto morfológico o ausente, por otro lado. Esta decisión está motivada por los porcentajes tan ajustados arrojados por el propio corpus sincrónico: en primer lugar, las construcciones transitivas con sujeto léxico o explícito registran $49 \%$ de casos; en segundo lugar, las construcciones transitivas con sujeto morfológico o ausente documentan $51 \%$ de casos. 
Los objetivos principales perseguidos en el presente apartado son: $i$ ) estudiar sincrónicamente la posición relativa del oD respecto del verbo transitivo en construcciones transitivas; ii) dar cuenta del grado de flexibilidad o estabilidad del orden vo en relación al orden ov; iii) determinar qué información pone el usuario del español en figura o en fondo ${ }^{15}$; y $i v$ ) estudiar si los tipos de prosa que guían el corpus inciden en el orden relativo de verbos transitivos y oD léxicos. Estos objetivos serán también tenidos en cuenta respecto de $\$ 5.2$ y $\$ 5.3$. Lo esperado es que el orden relativo entre el verbo transitivo y el od sea vo, con independencia de la presencia de sujetos léxicos o explícitos. A continuación, expongo en el cuadro 2 los datos obtenidos en el corpus.

\section{Cuadro 2}

Orden entre verbo transitivo y od léxico con sujeto léxico o explícito

$\begin{array}{lll} & \text { vo } & \text { ov } \\ \text { Bartleby } & 100 \%(33 / 33) & 0 \%(0 / 33) \\ \text { Bartra } & 100 \%(48 / 48) & 0 \%(0 / 48) \\ \text { Medicina } & 100 \%(35 / 35) & 0 \%(0 / 35) \\ \text { DLXXI } & 100 \%(23 / 23) & 0 \%(0 / 23) \\ & & \\ \text { PROMEDIO } & 100 \%(139 / 139) & 0 \%(0 / 139) \\ \text { N }=139 & & \end{array}$

En general, el análisis confirma con rotundidad que, según los datos consignados, el orden básico de constituyentes en construcciones transitivas del español actual es vo, con 100\% de casos. En la misma línea y de acuerdo con el cuadro 2 arriba, podemos dar cuenta de que la presencia de sujetos léxicos o explícitos no parece influir en la posición relativa que mantiene el oD léxico respecto del verbo transitivo. Esta información es muy significativa puesto que nos permite avanzar en la caracterización sintáctica de la oración transitiva del español actual como la oración con verbo transitivo y od léxico.

Respecto del género textual, el orden vo ha sido el único documentado, con $100 \%$ de casos, para los cuatro tipos de prosa analizados en el presente corpus; este dato parece confirmar que el orden de constituyentes en construcciones transitivas del español actual se inscribe dentro de la gramática nuclear, por lo que el tipo de prosa juega un papel secundario en cuanto a esta variable.

\subsection{El orden básico de constituyentes con sujeto morfológico}

En el presente apartado haré lo propio respecto de las construcciones transitivas del español actual con sujeto morfológico o ausente (8a-d). A continuación, presento algunos ejemplos paradigmáticos de construcciones transitivas con sujeto morfológico o ausente; marco el od léxito en negrita.

15 Según la Nueva gramática de la lengua española (RAE-ASALE 2009: \$40.1d), fondo es una de las denominaciones empleadas para la función de tema, y figura, una de las utilizadas para la función de rema. Estudiar exhaustivamente ambos conceptos excede los objetivos propuestos para el presente trabajo, dado que las funciones informativas constituyen un tema altamente problemático como argumentan varios autores (Gutiérrez Bravo 2008: 26; Puerma Bonilla 2018: 91-111). 
8a. Cuando no conocía la vida, escribía; ahora que conozco su significado, no tengo nada más que escribir [Bartleby, 46, xxi]

8b. Allí atrapó con sus dedos para siempre el fluido que la conectaba con el mundo [Bartra, 127, $\mathrm{xxI}]$

8c. por autoestima entendemos la evaluación que efectúa y mantiene comúnmente el individuo en referencia a sí mismo [Medicina, 16, xxI]

8d. y como ya no aguanté que me estuviera insultando agarré una piedra como de cantera, de las que utilizan para los cimientos, de un tamaño como de veinte centímetros de ancho, la cual estaba como a dos metros de distancia de donde estaban los pies de mi hijo [DLXXI, 26, XXI]

Tal y como observé en el apartado \$5.1, también en esta variable podemos apreciar que, en gran medida, la estructura interna de la frase nominal se caracteriza por un amplio arropamiento estructural, sobre todo, en extensiones al margen derecho del núcleo, en línea con lo expuesto por Company (2009: \$1.7). Este dato es significativo puesto que la mayor amplitud de la frase nominal del oD léxico parece que tiende a condicionar su posición posverbal en construcciones transitivas del español actual.

Lo esperado es que el orden relativo entre el verbo transitivo y el oD sea vo para los casos de oraciones transitivas con sujetos morfológicos o ausentes. A continuación, el cuadro 3 abajo arroja los datos consignados en el presente corpus sincrónico para esta variable.

\section{Cuadro 3}

Orden relativo entre verbo transitivo y oD léxico con sujeto morfológico o ausente

$\begin{array}{lll} & \text { vo } & \text { ov } \\ \text { Bartleby } & 100 \%(62 / 62) & 0 \%(0 / 62) \\ \text { Bartra } & 100 \%(35 / 35) & 0 \%(0 / 35) \\ \text { Medicina } & 100 \%(14 / 14) & 0 \%(0 / 14) \\ \text { DLXXI } & 97 \%(30 / 31) & 3 \%(1 / 31) \\ & & \\ \text { PROMEDIO } & 99 \%(141 / 142) & 1 \%(1 / 142) \\ \text { N }=142 & & \end{array}$

En términos generales, podemos resaltar dos informaciones especialmente relevantes: en primer lugar, las construcciones transitivas del español actual con sujeto morfológico o ausente se caracterizan por registrar en este corpus un rotundo orden básico de constituyentes vo, con 99\% de casos; en segundo lugar, el orden ov documenta solo un caso en la prosa jurídica, esto representa $1 \%$ de casos del corpus del español actual, y $3 \%$ de casos dentro de la prosa jurídica.

En relación con el género textual, las construcciones transitivas del español con sujeto morfológico o ausente evidencian que el tipo de prosa no es definitorio en el establecimiento del orden básico de constituyentes en construcciones transitivas del español actual. El único caso documentado de orden ov ocurre en construcciones transitivas con sujeto morfológico y ausente, en un texto jurídico. 


\subsection{Orden básico de constituyentes en construcciones transitivas del español actual}

En este apartado analizaré, a la luz de los resultados obtenidos en $\$ 5.1$ y $\$ 5.2$, el orden básico de constituyentes en construcciones transitivas del español actual con sujetos léxicos y morfológicos, toda vez que la presencia o ausencia del sujeto léxico no parece incidir, a la luz de la información contenida en este trabajo, en la disposición de los constituyentes argumentales. En suma, mostraré, de un lado, los casos en que el español actual se decanta por un orden de constituyentes de verbos transitivos vo (9a-d) con sujeto léxico o explícito, y (9e-h) con sujeto morfológico o ausente; de otro lado, aquellos otros casos en que el orden de constituyentes de verbos transitivos preferido es ov $(10 a)^{16}$. Incluyo a continuación ejemplos paradigmáticos; marco el sujeto en cursiva, el verbo en negrita y el oD en negrita y cursiva.

9a. A su muerte, un periódico parisino recordó muy oportunamente unas palabras de Wilde: «Cuando no conocía la vida, escribía; ahora que conozco su significado, no tengo nada más que escribir»

9b. Al igual que Humphrey Velmans, Edelman esquiva el asunto al que nos han enfrentado el autismo y los comportamientos antisociales [Bartra, 91, XXI]

9c. Los eventos críticos incrementan la tensión dentro del grupo familiar [Medicina, 37, xxI]

9d. el hoy occiso tuvo seis hermanos de nombres Alberto, José de Jesús, Daniel, Cecilia, Yesenia y Maibeline [DLXXI, 47, XXI]

9e. Otras investigaciones periodísticas descubrieron que tenía un cuarto nombre: Ret Marut, un escritor anarquista que había desaparecido en México en 1923 y los datos, pues, encajaban [Bartleby, 71, xxi]

9f. Veamos ahora, en ejemplos muy diferentes, la forma en que el uso de prótesis culturales ayuda a soportar situaciones patológicas dramáticas [Bartra, 129, XXI]

$9 \mathrm{~g}$. él detectó la necesidad de evaluar aspectos que no estaban relacionados y no eran evaluados en la historia clínica tradicional, puesto que ejercían una influencia positiva o negativa en el curso de la situación de salud o enfermedad que presentaba la persona en la consulta [Medicina, $39, \mathrm{xxI}]$

9h. y después de esto comenzó a hablar nuevamente y otra vez estuvo rayándome la madre, y como ya no aguanté que me estuviera insultando agarré una piedra como de cantera, de las que utilizan para los cimientos, de un tamaño como de veinte centímetros de ancho, la cual estaba como a dos metros de distancia de donde estaban los pies de mi hijo [DLXXI,26, XXI]

10a. me fui a mi casa con una botella de mezcal curada con refresco, pero al Toto ya no lo vi [DLXXI, 25, XXI]

La bibliografía examinada en este artículo da un lugar preferente al orden relativo vo, por lo que lo esperado es que sea este el orden dominante. A continuación, muestro en el cuadro 4 los datos obtenidos en el corpus sincrónico.

16 En el presente corpus sincrónico, existe el registro de un único caso de orden relativo entre verbo y oD léxico ov, prueba de que, aunque otros órdenes son posibles, estos parecen ser, de acuerdo con los datos del corpus, infrecuentes en el español actual escrito. 


\section{Cuadro 4}

Orden relativo entre verbo transitivo y oD léxico presente

$\begin{array}{lll} & \text { vo } & \text { ov } \\ \text { Bartleby } & 100 \%(95 / 95) & 0 \%(0 / 95) \\ \text { Bartra } & 100 \%(83 / 83) & 0 \%(0 / 83) \\ \text { Medicina } & 100 \%(49 / 49) & 0 \%(0 / 49) \\ \text { DLXXI } & 98 \%(53 / 54) & 2 \%(1 / 54) \\ & & \\ \text { PROMEDIO } & 100 \%(280 / 281) & 0 \%(1 / 281) \\ \text { N }=281 & & \end{array}$

En general, el corpus confirma con rotundidad que el orden relativo entre verbo transitivo y oD léxico u orden básico de constituyentes en construcciones transitivas sigue un orden no marcado vo, con $100 \%$ de casos, con independencia del género textual y de si existe o no copresencia de sujeto léxico. Cabe subrayar que he llevado a cabo esta investigación con base en el presente corpus y que los datos consignados en él confirman que el orden ov tiene una presencia residual; así, se ha documentado un único caso de orden ov a lo largo de las diversas calas realizadas para las cuatro obras analizadas. Además, es preciso tener en cuenta que, si bien la posición del od léxico es posverbal, el orden vo documentado en esta investigación es prácticamente el único documentado en todo el corpus.

Por otro lado, podemos notar que las necesidades pragmáticas del usuario del español actual parecen no incidir sobre la disposición del constituyente od léxico en las construcciones transitivas consignadas. En otras palabras, el orden sintáctico y pragmático parecen ser, en la actualidad, claramente rígidos, lo cual no quiere decir que el español no permita alterar el orden de los constituyentes sin dificultad, atendiendo a razones pragmáticas o extralingüísticas, sino que, de todas las combinatorias posibles, la canónica parece ser, de acuerdo con los datos consignados en el presente corpus, aquella en la que el od léxico se sitúa en una posición siempre posverbal, al menos en lo que respecta al español actual. En la misma línea y en relación al sujeto, Bogard (2015) justifica la escasa documentación del orden ov debido a que este constituyente parece constituir el "objeto de la comunicación", mientras que el od es "un comentario respecto de él o parte de él", en palabras del autor, el sujeto acapara el papel de “tópico", y el od léxico el de "comentario". Belloro (2012), por su parte, denomina a la información presupuesta, tópico, y a lo que se asevera sobre este, foco. En desacuerdo con esta información, Gutiérrez Bravo (2007) aclara que la posición inicial del sujeto no está motivada por requerimientos de caso o concordancia, ni por ser el sujeto un tópico por defecto.

\section{Conclusiones}

En la presente investigación, hemos estudiado el orden relativo entre verbo transitivo y od léxico u orden básico de constituyentes en construcciones transitivas del español actual. Estos parámetros de investigación no han sido tomados al azar ni fundados en estudios previos sobre orden de 
palabras u orden de constituyentes, sino que han sido informados por el análisis pormenorizado de las construcciones transitivas del español actual consignadas en un corpus compuesto de cuatro géneros textuales distintos, a saber: prosa literaria, prosa ensayística e historiográfica, prosa científica, y prosa jurídica. Así, los datos documentados sugieren que el español actual es ambivalente en cuanto a la presencia o ausencia de sujetos léxicos o explícitos; no obstante, las construcciones transitivas registradas prefieren, aunque de manera muy ajustada, sujetos morfológicos o ausentes en detrimento de sujetos léxicos o explícitos. En cuanto a su composición, el análisis de los datos confirma, por tanto, que verbo transitivo y od léxico son los elementos esenciales que caracterizan las construcciones transitivas prototípicas del español actual. Además, los datos obtenidos determinan que las construcciones transitivas con mayor número de sujetos léxicos o explícitos están documentadas en textos cuyo nivel de especialización es alto, mientras que en construcciones de carácter narrativo y creativo, el sujeto parece consabido, por lo que huelga la necesidad de expresarlo léxicamente.

Una vez caracterizada la construcción transitiva del español actual según los elementos esenciales que la componen y de acuerdo con los datos arrojados por el presente corpus, el análisis concluye que el español actual parece seguir un orden no marcado o básico (s)vo. En otras palabras, el oD léxico ocupa siempre una posición posverbal; por su parte, el sujeto léxico o explícito aparece en posición preverbal siempre que ocurre en la construcción transitiva, es decir, en poco menos de la mitad de los casos documentados.

Respecto del género textual, los datos sugieren que los cuatro tipos de prosa analizados parecen no condicionar la distribución de los constituyentes en oraciones transitivas del español actual, por lo que el orden de constituyentes parece adscribirse al ámbito de la gramática nuclear. No obstante, el único caso documentado de orden ov ocurre en un texto de prosa jurídica, en concreto, un caso que reproduce una declaración de los comparecientes en un proceso judicial.

En suma, los datos consignados en el presente corpus concluyen que el orden de constituyentes en construcciones transitivas del español actual se caracteriza por seguir un orden rígido en cuanto a la ocurrencia casi absoluta de un mismo orden de constituyentes (s)vo. Esta información refuta la hipótesis tradicional de que el español, a diferencia de otras lenguas como el inglés, posee gran flexibilidad respecto del orden de constituyentes. Es decir, esta investigación matiza la flexibilidad del español, puesto que esta se manifiesta en la posibilidad de alterar los diferentes órdenes de constituyentes alternativos al orden básico (s)vo por motivaciones extralingüísticas; sin embargo, esta posibilidad teórica queda restringida por la probabilidad sintáctica, es decir, por la casi exclusividad del orden (s)vo, de acuerdo con los datos consignados en un corpus compuesto de cuatro géneros textuales distintos, cuya misión ha sido favorecer la ocurrencia de motivaciones extralingüísticas, a saber: contextuales, pragmáticas, textuales y discursivas. 


\section{Referencias bibliográficas}

Bello, A. (1847-1860/1988). Gramática de la lengua castellana destinada al uso de los americanos, con las notas de Rufino José Cuervo. Madrid: Arco Libros.

Belloro, V. (2012). La estructura informativa. In R. Mairal, G. Lilián, \& C. González Vergara (Coords.), El funcionalismo en la teoría lingüística: la Gramática del Papel y la Referencia (pp. 225-244). Madrid: Akal.

Bentivoglio, P.; \& Weber, E. G. (1986). A functional approach to subject word order in spoken Spanish. In O. Jaeggli, \& C. Silva-Corvalán (Eds.), Studies in Romance Linguistics (pp. 3-40). Dordrecht: Foris.

Bogard, S. (2010). La frase nominal de objeto directo antepuesta al verbo en español. In S. Bogard (Ed.), Semántica, pragmática y prosodia: reflejos en el orden de palabras en español (pp. 69-115). México: El Colegio de México.

- (2015). Evolución de la estructura oracional con objeto directo antepuesto al verbo con especial atención al siglo XIX. Études romanes de Brno, 36 (2), 29-52.

Brucart, J. M.; \& Herranz, M. L. (1987). La sintaxis. Barcelona: Crítica.

Company, C. (1991). La frase sustantiva en español medieval. Cuatro cambios sintácticos. México: Universidad Nacional Autónoma de México.

- (2009). Estructura general de la frase nominal. In C. Company Company (Dir.), Sintaxis histórica de la lengua española. Segunda parte: La frase nominal (volumen 2) (pp. 3-56). México: FCE.

- (2016). Sintaxis histórica y tradiciones discursivas. El género textual como macrolocus del cambio sintáctico. In A. López Serena (Ed.), El español a través del tiempo. Estudios ofrecidos a Rafael Cano Aguilar (pp. 385-415). Sevilla: Publicaciones de la Universidad de Sevilla.

Comrie, B. (1981/1989). Word order. In Language universals and linguistic typology (pp. 86-103). Chicago: The University of Chicago Press.

Contreras, H. (1983). El orden de palabras en español. Madrid: Cátedra.

- (1991). On the position of subjects. Syntax and semantics, 25, 63-79.

Croft, W. (2007). Construction grammar. In D. Geeraerts, \& H. Cuyckens (Eds.), Cognitive Linguistics (pp. 463-508). Oxford: Oxford University Press.

Elvira, J. (2011). Constructions of uncontrolled state or event. The increase in productivity of a new argument structure in Old Spanish. Constructions and Frames, 3 (2), 184-207.

- (2012). Construcciones y significado: Aspectos diacrónicos de la transitividad en español. Corrientes de estudio en semántica y pragmática históricas. Madrid: Instituto Menéndez-Pidal.

—. (2015). El orden de palabras. In Lingüistica histórica y cambio gramatical (pp. 195-212). Madrid: Síntesis.

Fernández Soriano, O. (1993). Sobre el orden de palabras en español. Dicenda. Cuadernos de Filología Hispánica, 11, 113-152.

Goldberg, A. E. (1995). Constructions. A construction grammar approach to argument structure. Oxford: Oxford University Press.

Greenberg, J. H. (1963/1966). Some universals of grammar with particular reference to the order of meaningful elements. In Universals of Language (pp. 73-113). Cambridge/London: The M.I.T. Press.

Gutiérrez Bravo, R. (2008). La identificación de los tópicos y los focos. Nueva Revista de Filología Hispánica, 56 (2), 363-401. 
Lehmann, W. P. (1971). Why are OV languages agglutinative? In Linguistic Society of America Handbook (pp. 25-26).

. (1972). Contempory linguistics and IE studies. Publications of the Modern Language Association, 87, 976-993.

. (1973). A structural principle of language and its implications. Language, 49, 47-66.

. (1974). Proto-Indo-European syntax. Austin: University of Texas Press.

Luján, M.; Minaya, L.; \& Sankoff, D. (1984). The universal consistency hypothesis and the prediction of word order acquisition in the speech of bilingual children. Language, 60, 343-371.

Martín Butragueño, P. (1997). El papel de los factores sociales en el orden de palabras en español. In Varia lingüística y literaria. Tomo I: Lingüística (pp. 511-532). México: El Colegio de México.

Nebrija, A. (1492/2015). Gramática sobre la lengua castellana. México: Academia Mexicana de la Lengua.

Ocampo, F. (1990). The pragmatics of word order in constructions with a verb and a subject. Hispanic Linguistics, 4, 87-128.

Ocampo, F. A.; \& Klee, C. A. (1995). Spanish ov/vo word-order variation in Spanish-Quechua bilingual speakers. In C. Silva-Corvalán (ed.), Spanish in four continents: studies in language contact and bilingualism (pp.71-82). Washington D.C.: Georgetown University Press.

Ordóñez, F.; \& Treviño, E. (1999). Left dislocated subjects and the pro-drop parameter: A case study of Spanish. Lingua, 107, 39-68.

Puerma Bonilla, J. (2018). La función informativa del sujeto en construcciones transitivas en la historia del español. Revista de Historia de la Lengua Española, 13, 91-111.

Real Academia Española-Asociación de Academias de la Lengua Española. (2009). Nueva gramática de la lengua española. Madrid: Espasa Libros.

Schmidt, P. W. (1926). Die Sprachfamilien und Sprachenkreise der Erde. Heidelberg: Carl Winter.

Silva-Corvalán, C. (1984). Topicalización y pragmática en español. Revista Española de Lingüística, 14, 1-19.

Weil, H. (1844/1978). The order of words in the ancient languages compared with that of the modern languages. In Amsterdam Classics in Linguistics (vol. 14). Ámsterdam: John Benjamins.

\section{Corpus sincrónico}

[Bartleby] Vila-Matas, E. (2000). Bartleby y compañía. Barcelona: Anagrama.

[Bartra] Bartra, R. (2007). Antropología del cerebro. México: Fondo de Cultura Económica.

[Medicina] Compendio de tesis de medicina del siglo XXI.

[DLXXI]. Documentos jurídicos del siglo XXI. 
